\title{
ATUAÇÃO DO FARMACÊUTICO NO PROCESSO DE INTOXICAÇÃO POR ANALGÉSICOS NÃO-OPIÓIDES E ANTI-INFLAMATÓRIOS NÃO- ESTEROIDES (AINES)
}

\author{
Ana Carolina de Matos Egídio ${ }^{1}$ \\ Leonardo Guimarães de Andrade ${ }^{2}$ \\ Lívia Cabral Lobo ${ }^{3}$ \\ Michel Santos da Silva ${ }^{4}$
}

RESUMO: As intoxicações por medicamentos ocorrem por vários motivos, dentre os principais destaca-se, administração acidental ou intencional, na qual sucede a automedicação, erros de dosagem, desordem do medicamento. Portanto, existem vários tipos de intoxicação, deste modo será abordado os analgésicos não-opióides ou não narcóticos e anti-inflamatórios não esteróides (AINEs), que são o Paracetamol, Ibuprofeno e o Ácido acetilsalicílico (AAS). O objetivo principal é alertar a população sobre o uso indevido desses medicamentos isentos de prescrição, e que isso pode acarretar algumas complicações tóxicas. Sendo assim, é de extrema importância a atuação do farmacêutico nessas situações, pois, o profissional irá avaliar o consumo recente de uma determinada substância, as alterações clínicas que se desenvolvem com o uso dessa substância, e os sinais e sintomas específicos dessa intoxicação.

Palavra-chave: intoxicação; automedicação; medicamentos; Paracetamol; Ibuprofeno; Ácido acetilsalicílico.

ABSTRACT: Drug poisoning occurs for several reasons, among the main ones that stand out, accidental or intentional administration in which self-medication, dosing errors, drug disorder. Therefore, there are several types of intoxication in this way non-opioid or nonnarcotic analgesics and non-steroidal anti-inflammatory drugs will be addressed which are Paracetamol, Ibuprofen, and Acetylsalicylic Acid. The main objective is to alert the population about the misuse of these drugs without a prescription and may cause toxic complications. Therefore, the pharmacist must be active in these situations because the professional will evaluate the consumption of a pa rticular substance, the clinical changes that develop with the use of that substance, clinical changes that evolve with the custom of this substance, and the specific signs and symptom $\mathrm{s}$ of this intoxication.

\footnotetext{
I Graduação em Farmácia na Universidade Iguaçu. E-mail: carolzinhaegidio@hotmail.com

2 Mestre em Ciências do Meio Ambiente na Universidade Veiga de Almeida (2or6). Graduação em Enfermagem na Universidade Iguaçu

3 Mestre no programa de Ciências em Tecnologia de Processos Químicos e Bioquímicos pela Escola de Química da Universidade Federal do Rio de Janeiro (2012). Graduação em Farmácia pela Universidade Grande Rio, Rio de Janeiro (2004). Atualmente é docente no curso de Graduação em Farmácia na Universidade Iguaçu (desde 2013)no Estado do Rio de Janeiro. E-mail: livia.lobo@campusı.unig.br

4 Mestre em Ciências do Meio Ambiente na Universidade Veiga de Almeida (2016). Graduação em Ciências Biológicas pela Universidade Iguaçu (2009). Atualmente leciona como professor na Universidade Iguaçu, nos cursos de Graduação em Farmácia, Enfermagem e Engenharia Civil da Universidade Iguaçu no Estado do Rio de Janeiro.E-mail: michelbiodss@yahoo.com.br
} 
Keywords: intoxication, self-medication, Paracetamol, Ibuprofen, Acetylsalicylic Acid.

\section{INTRODUÇÃO}

Os processos de intoxicação humana têm-se transformado num dos mais graves problemas de saúde pública devido à falta de controlo e prevenção das intoxicações, associadas a um fácil acesso da população a um número crescente de substâncias (lícitas e ilícitas) com alto grau de toxicidade. "Os medicamentos são atualmente os principais agentes responsáveis por intoxicações agudas acidentais e intencionais" (FERREIRA, et al., 2008).

As intoxicações por medicamentos ocorrem por vários motivos, dentre os principais destaca: administração acidental ou intencional, isso acontece geralmente entre adolescentes e adultos na qual sucede a automedicação, erros de dosagem, desordem do medicamento e medicamentos ao alcance das crianças menores de 5 anos (FERREIRA, et al., 2008).

Portanto, existem vários tipos de intoxicação, deste modo será abordado os analgésicos não narcóticos e anti-inflamatórios não esteróides (AINES).

"Os analgésicos não opioides e anti-inflamatórios não esteróides (AINES) estãoentre os fármacos mais utilizados e prescritos no mundo, principalmente por terem efeito sintomático nas doenças ou processos inflamatórios em que estão indicados" (FARIAS, 2016). Além disso, são conhecidos como OTCs "over the counter", que significa sobre o balcão. Esses medicamentos não requerem prescrição e por isso a sua venda é livre (FARIAS, 2016).

No Brasil, essa dificuldade se estende ao acesso dos serviços de saúde, geralmente acontece com uma população vulnerável, e com isso, realiza a utilização desses fármacos. O seu objetivo é alertar a população que esse uso inapropriado pode acarretar algumas complicações indevidas ao organismo. Além disso, esses aspectos podem contribuir para o aumento de casos de óbitos por intoxicação medicamentosa (FARIAS, 2016).

“A utilização dos analgésicos, especificamente o paracetamol, são responsáveis por elevado número de registro de ocorrências da má administração de medicamentos" (FARIAS, 2016). A superdosagem desse fármaco pode provocar lesões hepáticas irreversíveis, podendo levar inclusive ao óbito. Essa utilização prolongada tem sido correlacionada à insuficiência renal (FARIAS, 20I6). 


\section{OBJETIVO GERAL}

É analisar o perfil das intoxicações de forma sucinta sobre o uso dos analgésicos não-opióides e anti-inflamatórios não-esteróides (AINES), com o objetivo de alertar a população que esse uso indevido pode acarretar algumas complicações tóxicas ao organismo e como o farmacêutico poderá intervir na prevenção.

\section{OBJETIVO ESPECÍFICOS}

- Analisar os perigos dos medicamentos que para a sociedade são inofensivos;

- Exemplificar as informações sobre a posologia, mecanismo de ação e o seu tratamento;

- Verificar casos de intoxicação medicamentosa;

- Apresentar os três medicamentos, que são: Paracetamol, Ibuprofeno, Ácido acetilsalicílico (AAS);

- Descrever atuação do farmacêutico na prevenção das intoxicações.

\section{METODOLOGIA}

A metodologia do trabalho será realizada com base em uma pesquisa descritiva na revisão bibliográfica dos autores. A triagem desse estudo foi realizada com o uso de base de dados encontrados em artigos científicos, revista científica, e em órgãos como o CRF (Conselho Regional de Farmácia) e Organização Mundial de Saúde.

O intuito dessa revisão de literatura é baseando-se na atuação do farmacêutico no processo de intoxicação por analgésicos não-opioides e anti-inflamatórios não-esteróides, que são de livre acesso nas farmácias.

Além disso, o desenvolvimento dessa pesquisa irá analisar os índices da automedicação, as tentativas de autoextermínio, superdosagem, as características de intoxicações, as etiologias, os diagnósticos de intoxicação por exposição de um ou mais agentes tóxicos e o papel do farmacêutico na orientação dessa prevenção.

\section{JUSTIFICATIVA}

O interesse por esse tema surgiu pela falta de dados e informações na sociedade. Além disso, participei na minha vivência acadêmica em hospital e drogarias, presenciando 
o uso contínuo desses medicamentos para conter os pacientes, seja de forma acidental ou intencional.

\section{O PROCESSO DE INTOXICAÇÃO}

Lembramos que, a toxicologia tem várias áreas distintas e com isso, iremos correlacionar com a toxicologia clínica que é um ramo de atuação para investigação de intoxicação. A intoxicação é designada pela absorção sistémica de uma ou mais substâncias tóxicas, onde se submete na quantidade e frequência de administração, necessária para que ocorra efeitos tóxicos no organismo (SILVA, 2017).

Deste modo, o autor ressaltar os dados da Organização Mundial de Saúde, que diz assim:

Organização Mundial de Saúde (OMS), em 2012, a nível mundial cerca de 193460 pessoas morreram por intoxicação não intencional. A este número de mortes estão associadas diversas causas, sendo as mais comuns: os pesticidas, produtos químicos domésticos, monóxido de carbono e os medicamentos. As intoxicações acidentais são responsáveis pela perda de mais de 10,4 milhões de anos de vida saudável, embora cerca de um milhão de pessoas morra a cada ano por suicídio, onde os produtos químicos são a principal fonte dessas intoxicações intencionais (ZUCOLOTO, A., et al., 2017)

Entendemos que, "a intoxicação é um problema de Saúde Pública de importância global' (ZUCOLOTO, A., et al., 2017). No Brasil, temos o Sistema de Informação de Agravos de Notificação (SINAN) onde ficam os casos registrados dos pacientes e outras particularidades. Para obter as informações do paciente é necessário fazer uma triagem para saber se esse utente tem alguma alteração de comportamento e do estado mental principalmente e se caso o paciente não estiver consciente, o ideal é buscar essas informações com os familiares presentes no local (ZUCOLOTO, A., et al., 2017).

Neste caso, o profissional responsável irá verificar os seguintes critérios: consumo recente de uma determinada substância, as alterações clínicas que se desenvolvem com o uso dessa substância, e os sinais específicos dessa intoxicação. É a partir dessas intoxicações que irei descrever o meu trabalho, pondo em evidência, a atuação do farmacêutico no processo de intoxicação por analgésicos não-opióides e anti-inflamatórios não-esteróides (SILVA,2017). 


\section{INTOXICAÇÃO POR ANALGÉSICOS}

Consiste em uma série de sinais e sintomas produzidos, quando um medicamento é ingerido, inalado, injetado ou entra em contato com a pele, olhos ou mucosas. Sendo assim, é impossível classificar que todas as substâncias são seguras ou tóxicas (SILVA, 20I7; GRETZLER, V., et al., 2018).

Além disso, a prescrição de analgesia torna-se fundamental não só por razões humanitárias, mas também para diminuir as complicações associadas à dor, a mobilidade, diminuindo os internamentos, os estados de delírio e capacitando uma melhora na qualidade de vida. Dentro da analgesia, o ópio é a substância natural mais potente no manejo da dor. Por isso, que no Brasil temos o Sistema Nacional de Informações Tóxico-farmacológicas (Sinitox) que foi elabora pelo Ministério da Saúde e que hoje é vinculada na Fundação Oswaldo Cruz (ZUCOLOTO, A., et al., 2017; GRETZLER, V., et al., 2018).

Dentro deste tema, no decorrer dessa revisão bibliográfica, será abordado de forma detalhada as intoxicações ocorridas por medicamentos com ação analgésica, não-opióides e anti-inflamatórios não esteroidal (AINES), mais consumidos no Brasil, nomeados, como paracetamol, ibuprofeno e o ácido acetilsalicílico (AAS).

\section{PARACETAMOL}

O paracetamol pertence à classe dos analgésicos e antipiréticos, tendo média ação analgésica e alta ação antipirética (FREITAS, et al., 2017). Geralmente, "o paracetamol é o analgésico oral de primeira escolha para ser utilizado por longos períodos, no tratamento sintomático de dor leve a moderada, como acontece na osteoartrite, nas dores musculares ou tendinosas" (SILVA, 2017).

No entanto, a sua popularidade é atribuída devido a sua eficácia, segurança e à sua tolerabilidade aos efeitos adversos quando é administrado corretamente nas doses terapêuticas recomendadas. Além disso, o paracetamol tem várias formulações incluindo comprimidos, comprimidos efervescentes, comprimidos de libertação prolongada, xarope, supositórios direcionados para as crianças e as preparações intravenosas, podendo ser administradas por via oral, retal e endovenosa (SILVA, 2017). 
Geralmente, as reações adversas associadas à utilização de paracetamol são raras. No entanto, se isso acontecer pode causar vômitos ou náuseas, obstipação e pirose conhecido como azia (KOPPEN, et al., 2014).

Deste modo, "o mecanismo de ação do paracetamol é rapidamente absorvido a partir do trato gastrointestinal (TGI) com pico de concentrações atingidas em 90 minutos para uma dose terapêutica" (FREITAS, et al., 2017). A sua biodisponibilidade é de 6o a 95 \%, portanto, é rápida distribuição, com um volume de $0,9 \mathrm{~L} / \mathrm{kg}$ e ligação mínima a proteínas plasmáticas nas concentrações terapêuticas, podendo chegar a 50\% em casos de overdose. Além disso, o tempo de meia-vida do paracetamol é de 2,0 a 2,5 horas, mas quando há lesão hepática, a meia-vida é prolongada para mais de 4 horas (SILVA, 2017).

Sendo assim, a oxidação do paracetamol é por via citocromo $\mathrm{P}_{450}$ (CYP450), responsável por 5 a $15 \%$ desse metabolismo. E com isso, temos outras vias como citocromos $\mathrm{P}_{450}\left(\mathrm{CYP}_{2} \mathrm{E}_{\mathrm{I}}\right)$ e a $\left(\mathrm{CYP}_{3} \mathrm{~A}_{4}\right)$, resultando na formação de um metabolito intermediário reativo, o N-acetil-p-aminobenzoquinonimina (NAPQI), que é responsável pela hepatotoxicidade do fármaco (KOPPEN, et al., 2014; SILVA, 2017).

No entanto, em caso de sobredosagem, a capacidade das principais vias de metabolização é excedida, e por conseguinte é produzido uma maior proporção de NAPQI. No caso, de acúmulo de NAPQI pode provocar uma necrose hepatocelular, o que pode conduzir ao transplante hepático ou até mesmo o óbito (KOPPEN, et al., 2014)

A posologia do paracetamol tem início is a 30 minutos após a administração oral e permanece por um período de 4 a 6 horas. Em casos de administração por via oral (VO) em crianças solicita cuidados especiais e doses adequadas. Terapeuticamente, para os adultos é recomendada a administração oral de doses compreendidas entre 325 e $650 \mathrm{mg}$ a cada 4-6h se necessário, ou administra a única de Iooomg, 3-4 vezes por dia (SILVA, 2017). Sendo assim, a dose máxima diária é de $4 \mathrm{~g}$. Em casos, do paciente sofrer de uma doença hepática, dependência de álcool, precisa passar por um processo prolongado de jejum. Vale ressaltar, que a dose diária não deverá ultrapassar de 3g. Em situações de superdosagem, as primeiras manifestações que ocorrem são náuseas ou vómitos e dores fortes no abdómen (KOPPEN, et al., 2014; SILVA, 2017).

O tratamento de intoxicações por paracetamol é através da administração por via oral (V.O) ou intravenosa (I.V) de N-acetilcisteína (NAC), e o antídoto que é capaz de 
restabelecer os níveis de glutationa hepática, através do fortalecimento da cisteína (SILVA, 2017).

\section{IBUPROFENO}

O ibuprofeno é um anti-inflamatório, na qual pertence à classe terapêutica dos (AINEs). "Mundialmente é das substâncias ativas mais consumidas pelas suas propriedades anti-inflamatórias, analgésicas e antipiréticas" (SILVA, 2017). O ibuprofeno é indicado no tratamento de dor ligeira a moderada, dor de cabeça ou enxaqueca, dor associada à dismenorreia, dor pós-operatório, e com isso, aliviando os sintomas associados as doenças reumáticas, como artrite reumatoide e osteoartrite. Sendo assim, os seus efeitos dependem das doses, e poderão ser reduzidos com a administração da menor dose, portanto, a sua eficácia durante o período de tratamento será menor (MATHIAS, 2020).

É indicado para adultos a administração oral de doses entre 200 e 80omg, no intervalo de 4 a 6 horas, no período de 7 dias. Em situações de ação analgésicas e antipiréticas, a dose recomendada é de 40omg, no intervalo de 4 a 6 horas (SILVA, 2017; MATHIAS, 2020). Veja na tabela a seguir:

Tabela r: Posologia do ibuprofeno em crianças.

\begin{tabular}{|c|c|c|}
\hline Tratamento & $\begin{array}{c}\text { Peso }(\mathrm{Kg}) / \mathrm{Idade} \\
\text { (anos) }\end{array}$ & Posologia \\
\hline \multirow[t]{4}{*}{ Dor e/ou febre } & $>7 \mathrm{Kg}_{\mathrm{g}}$ & $\begin{array}{c}20230 \mathrm{mg} / \mathrm{Kg} / \mathrm{dis} \text {, repetindo em } 3- \\
4 \times / \text { diária }\end{array}$ \\
\hline & $x-2 \operatorname{anos}$ & $50 \mathrm{mg}, 3-4 x / \mathrm{dia}$ \\
\hline & 3-7 anos & $100 \mathrm{mg}, 3-4 x / \mathrm{dis}$ \\
\hline & 8- -12 anos & $200 \mathrm{mg}, 3-4 x / \mathrm{dia}$ \\
\hline $\begin{array}{c}\text { Artrite crônica } \\
\text { juvenil }\end{array}$ & $>7 \mathrm{Kg}$ & $\begin{array}{c}30 \Omega 40 \mathrm{mg} / \mathrm{Kg}_{\mathrm{g}} / \mathrm{dis} \text {, repetindo em } 3^{-} \\
4 \times / \text { diária }\end{array}$ \\
\hline
\end{tabular}

Fonte: SILVA, 2017

O ibuprofeno em alguns casos de intoxicação, ocorre pela inibição excessiva da COX-ı e, consequentemente, uma redução da síntese de prostaglandinas. Desta forma, a inibição excessiva da $\mathrm{COX}_{-1}^{-1}$ pode resultar anomalias hematológicas, nomeadamente a alteração do tempo de protrombina afetando a agregação plaquetária. É viável ter uma atenção especial aos pacientes na avaliação terapêutica com anticoagulantes ou 
antiplaquetárias, visto que, em pacientes idosos, há um aumento da frequência das reações adversas aos anti-inflamatórios (AINEs), especialmente hemorragia e perfuração, que podem ser fatais (SILVA, 2017; MATHIAS, 2020).

Normalmente, o tratamento de ingestão de pequenas quantidades se manifesta no trato gastrointestinal verificar a presença de alguns fármacos como paracetamol e ácido acetilsalicílico (AAS) no sangue podendo influenciar na gravidade da toxicidade (SILVA, 2017).

\section{ÁCIDO ACETILSALICÍLICO (AAS)}

O ácido acetilsalicílico pertence ao grupo dos fármacos anti-inflamatórios nãoesteroidais e tem as suas propriedades ativas que são anti-inflamatório, analgésico, antipirético. Terapeuticamente, é indicado para o tratamento sintomático de dor ligeira a moderada, de pirexia e de doenças inflamatórias, como artrite reumatoide e a doença de Kawasaki (SILVA, 2017). Essa doença é rara e acontece, geralmente, em crianças e seus sintomas são febres persistentes (SILVA, 2017).

Desta forma, é administrada por via oral (V.O) e o seu mecanismo procede na inibição irreversível da atividade das isoenzimas cicloxigenase COX I e COX 2. Seu tempo de ação persiste por aproximadamente ı dias, que é o tempo de meia-vida das plaquetas, podendo assim ser ingerida uma vez ao dia. Após a ingestão, será absorvido pelo estômago e seu início de ação entre 20 e 30 minutos. Com isso, após a absorção são encontrados no sangue, na saliva, no líquido sinovial e cefalorraquidiano e na bile (SILVA, 2017). Consequentemente, no sangue sofre o processo de hidrólise com ácido acético e ácido salicílico, ou seja, seu tempo de meia-vida de varia de 2 a 3 horas, sendo excretados pelos rins. Além disso, os principais efeitos de uma reação adversa são os distúrbios do trato gastrointestinal superior e inferior, sangramentos, distúrbios renais e reação de hipersensibilidade (SILVA, 2017).

O ácido acetilsalicílico é conhecido popularmente como aspirina, AAS e salicilatos é indicado para adolescente e idosos na faixa etária de $16-65$ anos. Em doses terapêuticas é recomendado entre 500 e $1000 \mathrm{mg}$, ou seja, não podendo ingerir mais de 3 comprimidos por dia e o AAS infantil é de roo mg, um comprimido por dia. É importante destacar que isso pode variar de acordo com avaliação e prescrição médica (SILVA, 2017; JONSSON, 202I). 
A venda desse medicamento é com ou sem prescrição médica, com isso, a facilidade desse salicilato é bastante extensa, portanto, essa substância atingir de forma intencional ou acidental os valores tóxicos. Neste caso, se o adulto ingerir doses de io a $30 g$ de aspirina provocará uma intoxicação grave que poderá ter resultados letais, e isso acontece em crianças com ingestão de $3 g$ de aspirina. Desta forma, as intoxicações se diferenciam em leve, moderado e grave (SILVA, 2017; JONSSON, 2021).

O tratamento com carvão ativado, diurese alcalina com cloreto de potássio, e a hemodiálise que é o tratamento mais eficaz na eliminação de salicilatos bem como na correção dos distúrbios ácido-base associadas à intoxicação grave por AAS (SILVA, 2017).

\section{ATUAÇÃO DO FARMACÊUTICO NA PREVENÇÃO DAS INTOXICAÇÕES}

Sabemos que, o farmacêutico é crucial nessas intervenções medicamentosas, ou seja, um dos dados de extrema importância é a ocorrência numerosa de erros de administração do medicamento (GRETZLER, V., et al., 2018).

Portanto, os medicamentos são as substâncias que mais causam intoxicação no Brasil, sendo assim, o profissional farmacêutico além de orientar essas ações medicamentosas, identifica e reduz os ricos associados as ações terapêuticas. Com isso, irá intervir nessa prática de automedicação. Nesses casos, a interação farmacêutica é primordial pois na prática isso afeta demais o quadro clínico do paciente (GRETZLER, V., et al., 2018).

Segundo o autor (FALVO, et al., 2017), que diz assim com evitar as intoxicações, seguindo as orientações:

- Informe-se com o farmacêutico quanto às doses e o modo de administrar;

- Leia a bula;

- Não estimule as crianças dizendo que o medicamento é bom, doce ou faz crescer;

- Armazene os medicamentos de preferência em armários que possam ser fechados;

- Não misture medicamentos diferentes na mesma embalagem;

- Observe cuidadosamente o rótulo do medicamento antes de administrar;

- Dispensar o medicamento de forma segura;

- Não dê medicamentos no escuro. 


\section{DISCUSSÃO}

De acordo com dados abordados nesse trabalho, entendemos que, o uso inapropriado do medicamento pode acarretar diversas reações, ou seja, isso acontece com os medicamentos isento de prescrição conhecido como os (MIPs). Sendo que, hoje em dia é bastante comum a compra e venda, e é uma discussão favorável e de se pensar, essa questão do livre acesso. Por mais que seja influenciável na indústria farmacêutica, a população busca uma cura ou prevenção de uma forma erronia, na intenção de uma possível melhora no quadro clínico e que se for utilizado de forma indesejada isso pode ser excêntrico à saúde.

\section{CONCLUSÃO}

O tema abordado é de extrema importância, pois alerta a sociedade que a intoxicação por esses medicamentos acontece de forma acidental ou intencional, ressaltando assim, os casos com os idosos e as crianças, e que infelizmente é um dos maiores problemas de saúde pública decorrente pela falta de informação.

Concluímos que, o profissional farmacêutico além de fazer as atividades relacionadas da dispensação de medicamentos, irá orientar e prevenir essas automedicações e com isso, passa a ter uma relevância maior no contato direto com os utentes de maneira que seja acolhedora exercendo a sua competência com a farmacoterapia.

\section{REFERÊNCIAS}

CORUJA, C.; Intoxicação por paracetamol no Rio Grande do Sul. Porto Alegre: Universidade Federal do Rio Grande do Sul, 2012. p.40. Trabalho de Conclusão de Especialização em Saúde Pública.

FALVO, A. et al.; Manual de orientação ao farmacêutico: aspectos legais da dispensação. Conselho Regional de Farmácia do Estado de São Paulo. Disponível em: < http://www.crfsp.org.br/documentos/materiaistecnicos/Aspectos_Legais_da_Dispensaca o>. Acesso em: 20 de setembro de 2021. 
FARIAS, P.; Aspectos epidemiológicos das intoxicações por analgésicos não opioides e anti-inflamatórios não esteroides em um hospital de urgência e emergência da rede pública do Brasil. Revista Medicina Minas Gerais, Belo Horizonte, v.26, n.5, p.II-I4, 2016.

FERREIRA, A.; Avaliação da Contribuição dos Medicamentos nas Intoxicações Agudas em Portugal. Revista da Faculdade de Ciências da Saúde, Portugal, n.5, p.94-ıio, 2008.

FREITAS, J. A. B. et al.; Medicamentos isentos de prescrição: perfil de consumo e os riscos tóxicos do paracetamol. Revista Intertox de Toxicologia Risco Ambiental e Sociedade, Ceará, v.10, n.3, p.134-154, out. 2017.

GRETZLER, V. S. et al.; Atuação do farmacêutico no urm e na prevenção de intoxicação medicamentosa. Revista Científica FAEMA: Revista da Faculdade de Educação e Meio Ambiente, Rondônia, v.9, n. ed esp, p.547-550, maio/jun, 2018.

JONSSON, R. Bula do AAS Protect. Disponível em: < https://consultaremedios.com.br/aas-protect/bula>. Acesso em: I8 de set. de 2021.

MATHiAS, F. T.; Bula do Ibuprofeno Comprimido Cimed. Disponível em: $\langle$ https://consultaremedios.com.br/ibuprofeno-comprimido-cimed/bula〉. Acesso em: I9 de set. de 2021.

KOPPEN,

A.

et

al.;

Recommendations

the paracetamol treatment nomogram and side effects of $\mathrm{N}$-acetylcysteine. Article in The Netherlands Journal of Medicine, v.72, n.5, p.25I-257, jun. 2014.

SILVA, M. I. M.; Intoxicações por analgésicos e qual o papel do farmacêutico na prevenção destas. 2017. p.99. Dissertação (Mestrado Integrado em Ciências Farmacêuticas), Universidade Egas Moniz, Portugal, 2017.

ZUCOLOTO, A. D. et al.; Manual de toxicologia clínica: Orientações para assistência e vigilância das intoxicações agudas. Disponível em: < http://www.cvs.saude.sp.gov.br/ler.asp?nt_codigo $=1592 \& n t \_t i p o=0 \& t e \_c o d i g o=40>$. Acesso em: 20 de setembro de 202I. 